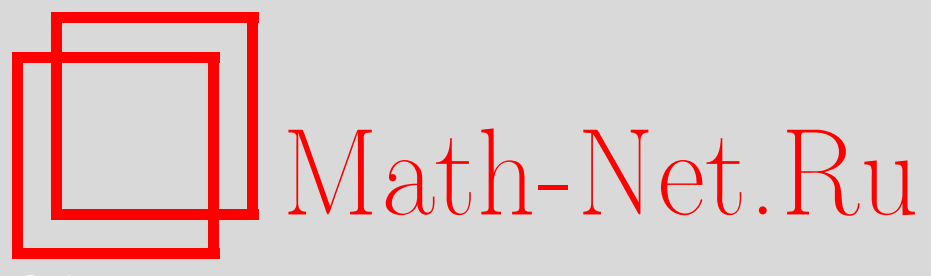

В. В. Рыжиков, А. Е. Троицкая, Тензорный корень из изоморфизма и слабые пределы преобразований, $M a$ тем. заметки, 2006, том 80, выпуск 4, 596-600

DOI: https://doi.org/10.4213/mzm2852

Использование Общероссийского математического портала Math-Net.Ru подразумевает, что вы прочитали и согласны с пользовательским соглашением http://www . mathnet.ru/rus/agreement

Параметры загрузки:

IP : 54.162 .85 .209

26 апреля 2023 г., 05:22:37

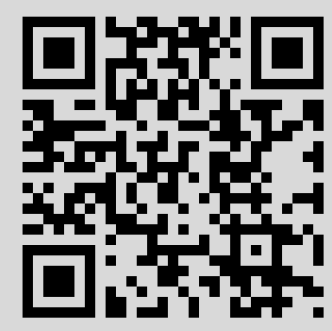




\section{ТЕНЗОРНЫЙ КОРЕНЬ ИЗ ИЗОМОРФИЗМА \\ И СЛАБЫЕ ПРЕДЕЛЫ ПРЕОБРАЗОВАНИЙ}

\section{В. В. Рыжиков, А. Е. Троицкая}

Доказано, что преобразования $S$ и $T$ изоморфны, если изоморфны их декартовы квадраты, при условии, что для преобразования $T$ некоторая последовательность степеней слабо сходится к полиному.

Библиография: 7 названий.

Пусть $S$ и $T$ - сохраняющие меру обратимые преобразования вероятностного пространства Лебега $(X, \mu)$ (как говорят, автоморфизмы пространства $(X, \mu))$. Их декартовы квадраты $S \times S$ и $T \times T$ метрически изоморфны, если выполнено

$$
J(S \times S) J^{-1}=T \times T,
$$

где $J$ - автоморфизм пространства $(X \times X, \mu \times \mu)$. Сюжет заметки связан с вопросом: изоморфны ли преобразования $S$ и $T$, если изоморфны их декартовы степени? Вопрос поставлен Ж.-П. Тувено (J.-P. Thouvenot). Контрпримеры неизвестны. Сложность задачи объясняется трудностью описания факторов декартовых степеней автоморфизмов. В заметке рассмотрен случай, когда полугруппа слабых пределов степеней автоморфизма содержит полином. Это свойство нашло приложения в теории самоприсоединений и в спектральной теории (см., например, [1]-[4]). Ряд задач эргодической теории был решен методом категорий, когда доказывалась типичность изучаемого свойства динамической системы. Недавно возрос интерес к типичным свойствам, и этот интерес объясняется обнаружением, можно сказать, необычных типичных явлений в эргодической теории (см. недавнюю работу [5] и ссылки на статьи других авторов). Настоящая заметка косвенно примыкает к этой тематике, так как в ней рассматривается такое типичное свойство автоморфизма $T$ как наличие полинома $\mathscr{P}(T)$ в полугруппе слабых пределов его степеней.

Будем обозначать теми же символами, что и автоморфизмы, унитарные операторы, им отвечающие. Полиномом называется ряд вида

$$
\mathscr{P}(T)=a \Theta+\sum_{i} a_{i} T^{i}
$$

где $i \in \mathbb{Z}, a_{i} \geqslant 0, a+\sum_{i} a_{i}=1, \Theta$ - ортопроекция в $L_{2}(X, \mu)$ на пространство констант.

Работа первого автора выполнена при финансовой поддержке программы "Ведущие научные школы", грант № НШ-457.2003.1.

(C) В. В. Рыжиков, А.Е. ТроицкАя, 2006 
СоглАшениЕ. Ниже обозначение $\mathscr{P}(T)$ используется для полиномов, которые содержат хотя бы два ненулевых члена (не являются мономами).

Теорема. Пусть для слабо перемешивающего автоморфизма $T$ и некоторой последовательности $n_{k} \rightarrow \infty$ имеет место слабая сходимость $T^{n_{k}} \rightarrow \mathscr{P}(T)$. Если степени $S \times \cdots \times S$ (d сомножителей) $u T \times \cdots \times T$ (d сомножителей) изоморфнь, то автоморфизмы $S$ и т изоморфны.

Частный случай этой теоремы, когда $\mathscr{P}(T)=a I+(1-a) T$, был получен А. Е. Троицкой в курсовой работе 2004 г. Другой частный случай $\mathscr{P}(T)=a \Theta+(1-a) I$ рассмотрен в [6].

Предварительно докажем две леммы.

Лемма 1. Если выполнены условия теоремъ, то $S^{n_{k}} \rightarrow \mathscr{P}(S)$.

ДокАЗАтЕЛьство. Пусть (при $d=2$ ) имеет место равенство

$$
J^{-1}(S \otimes S) J=(T \otimes T) .
$$

Получим

$$
J^{-1}(S \otimes S)^{n_{k}} J=(T \otimes T)^{n_{k}} \rightarrow \mathscr{P}(T) \otimes \mathscr{P}(T) .
$$

Считаем, что выполнено условие

$$
S^{n_{k}} \rightarrow Q
$$

для некоторого оператора $Q$ (это обеспечивается выбором подпоследовательности из $n_{k}$ - ее и рассмотрим). Переходя к слабому пределу, получим

$$
Q \otimes Q=J \mathscr{P}(T) \otimes \mathscr{P}(T) J^{-1} .
$$

Обозначим $A \geqslant B$ ( $A, B$ - операторы), если для любой измеримой ограниченной неотрицательной функции $f$ выполнено $A f \geqslant B f$. Так как

$$
\mathscr{P}(T) \otimes \mathscr{P}(T) \geqslant a_{i}^{2}(T \otimes T)^{i},
$$

то выполнено

$$
Q \otimes Q \geqslant a_{i}^{2} J(T \otimes T)^{i} J^{-1} \geqslant a_{i}^{2}(S \otimes S)^{i},
$$

следовательно, для всех $i$ имеет место неравенство

$$
Q \geqslant a_{i} S^{i}
$$

Так как выполнено

$$
\mathscr{P}(T) \otimes \mathscr{P}(T) \geqslant a^{2} \Theta \otimes \Theta
$$

получим

$$
Q \otimes Q \geqslant a^{2} \Theta \otimes \Theta, \quad Q \geqslant a \Theta .
$$

Таким образом, $Q \geqslant \mathscr{P}(S)$, что в силу равенства $Q \mathbf{1}=\mathscr{P}(S) \mathbf{1}(\mathbf{1}$ - функция, тождественно равная 1$)$ приводит к равенству $Q=\mathscr{P}(S)$. Случай $d>2$ рассматривается аналогично. 
Лемма 2. Пусть $\left\{P_{v}\right\}$ u $\left\{R_{w}\right\}-$ наборы автоморфизмов, коммутирующих с эргодическим автоморфизмом $U$. Если

$$
\sum_{v} a_{v} P_{v}=\sum_{w} b_{w} R_{w}
$$

все $P_{v}$ различны и все $R_{w}$ различны, а веса $a_{v}, b_{w}$ положительны, то для некоторой $\sigma$ (перестановки индексов) выполнено

$$
P_{v}=R_{\sigma(v)}, \quad a_{v}=b_{\sigma(v)} .
$$

ДоказАтельство. На графике автоморфизма $P_{v}$ сосредоточена мера $\nu_{v}, \nu_{v}(Y)=$ $\mu\left(\left\{x:\left(x, P_{v} x\right) \in Y\right\}\right)$. Определим аналогично меру $\eta_{w}$, отвечающую автоморфизму $R_{w}$. Из равенства

$$
\sum_{v} a_{v} P_{v}=\sum_{w} b_{w} R_{w}
$$

вытекает равенство

$$
\sum_{v} a_{v} \nu_{v}=\sum_{w} b_{w} \eta_{w}
$$

Меры $\nu_{v}, \eta_{w}$ инвариантны и, более того, эргодичны относительно $U \otimes U$. Различным индексам $v$ отвечают различные меры (аналогично для индексов $w$ ) - это следствие того, что два автоморфизма $P, P^{\prime}$, коммутирующих с эргодическим действием и совпадающих на множестве положительной меры, совпадают почти всюду. Действительно, равенство $P x=P^{\prime} x$ влечет за собой $U^{n} P x=U^{n} P^{\prime} x, P U^{n} x=P^{\prime} U^{n} x$, т.е. $P, P^{\prime}$ совпадают на $U$-инвариантном множестве.

Каждая из сумм в равенстве

$$
\sum_{v} a_{v} \nu_{v}=\sum_{w} b_{w} \eta_{w}
$$

есть разложение на эргодические компоненты инвариантной меры. Из единственности такого разложения вытекает $\nu_{v}=\eta_{\sigma(v)}$, что равносильно утверждению леммы.

ДоказАтеЛЬСтво теоремы. Случай $d=2$. Наша цель - показать, что изоморфизм $J$ переводит координатную алгебру в координатную. Тем самым, будет установлен изоморфизм между $S$ и $T$. Для этого в нашем случае достаточно установить, что образ пространства $1 \otimes L_{2}(X, \mu)$ под действием $J$ совпадает с пространством $1 \otimes L_{2}(X, \mu)$ или $L_{2}(X, \mu) \otimes \mathbf{1}$.

Имеем

$$
J(T \otimes T)^{n_{k}} J^{-1}=(S \otimes S)^{n_{k}} .
$$

Переходя к слабому пределу, получим

$$
J(\mathscr{P}(T) \otimes \mathscr{P}(T)) J^{-1}=\mathscr{P}(S) \otimes \mathscr{P}(S) .
$$

Перепишем равенство в следующем виде:

$$
\sum_{i, j} a_{i} a_{j} J\left(T^{i} \otimes T^{j}\right) J^{-1}+V=\sum_{i^{\prime}, j^{\prime}} a_{i^{\prime}} a_{j^{\prime}} S^{i^{\prime}} \otimes S^{j^{\prime}}+V^{\prime},
$$

где оператор $V$ является линейной комбинацией марковских операторов вида $J\left(T^{i} \otimes\right.$ $\Theta) J^{-1}$ и $J\left(\Theta \otimes T^{j}\right) J^{-1}$, а оператор $V^{\prime}$ - линейной комбинацией марковских операторов вида $S^{i^{\prime}} \otimes \Theta$ и $\Theta \otimes S^{j^{\prime}}$. Марковскому оператору $V: L_{2} \otimes L_{2} \rightarrow L_{2} \otimes L_{2}$ отвечает 
мера $\nu$ на пространстве $(X \times X) \times(X \times X)$ (полиморфизм в терминологии Вершика [7]). Оператору $V^{\prime}$ отвечает мера $\nu^{\prime}$. Мера $\Delta_{R}$, сосредоточенная на графике автоморфизма $R$ пространства $(X \times X, \mu \times \mu)$, взаимно сингулярна с мерой $\nu$ (и мерой $\left.\nu^{\prime}\right)$. Действительно, при разложении меры $\nu$ в систему координатных условных мер получим, что эти условные меры непрерывны, в то время как условные меры для $\Delta_{R}$ суть одноточечные меры. Таким образом, получим, что $\nu=\nu^{\prime}$, следовательно, $V=V^{\prime}$, поэтому

$$
\sum_{i, j} a_{i} a_{j} J\left(T^{i} \otimes T^{j}\right) J^{-1}=\sum_{i^{\prime}, j^{\prime}} a_{i^{\prime}} a_{j^{\prime}} S^{i^{\prime}} \otimes S^{j^{\prime}} .
$$

Пусть найдется пара $i, j$ такая, что $i \neq j, a_{i}>0, a_{j}>0$. Применяя лемму 2 , получим

$$
J\left(T^{i} \otimes T^{j}\right) J^{-1}=S^{i^{\prime}} \otimes S^{j^{\prime}}, \quad J\left(I \otimes T^{j-i}\right) J^{-1}=S^{i^{\prime}-i} \otimes S^{j^{\prime}-i} .
$$

Пространство $L_{2} \otimes \mathbf{1}$ является неподвижным для оператора $I \otimes T^{j-i}$, поэтому пространство $J\left(L_{2} \otimes \mathbf{1}\right)$ - неподвижное для оператора $S^{i^{\prime}-i} \otimes S^{j^{\prime}-i}$. Очевидно, что роль $J\left(L_{2} \otimes \mathbf{1}\right)$ могут играть только пространство $L_{2} \otimes \mathbf{1}$ (когда $i^{\prime}=i$ ) или пространство $\mathbf{1} \otimes L_{2}\left(\right.$ когда $\left.j^{\prime}=i\right)$, причем одна из возможностей выполняется обязательно. Таким образом, изоморфизм $J$ отображает пространство $L_{2} \otimes \mathbf{1}$ в себя или в $\mathbf{1} \otimes L_{2}$. Но это означает, что координатный $T$-фактор изоморфно отображается в координатный $S$-фактор, т.е. $T$ и $S$ изоморфны.

Если нужной пары $i, j$ нет, то в ряде $\mathscr{P}(T)$ только для одного индекса $i$ выполнено $a_{i}>0$ (кроме того, $a>0$ ). Если вместо последовательности $n_{k}$ рассмотреть $n_{k}-i$, получим

$$
T^{n_{k}-i} \rightarrow a \Theta T^{-i}+a_{0} I=a \Theta+a_{0} I .
$$

Мы оказались в ситуации, которая была рассмотрена в работе [6].

Отличие случая $d>2$ от случая $d=2$ состоит в следующем. Рассматривая уравнение

$$
J\left(T^{i} \otimes T^{j} \otimes \cdots \otimes T^{k}\right) J^{-1}=S^{i^{\prime}} \otimes S^{j^{\prime}} \otimes \cdots \otimes S^{k^{\prime}},
$$

которое мы получаем при условии $i \neq j, \ldots, k$, и преобразуя его к виду

$$
J\left(I \otimes T^{j-i} \otimes \cdots \otimes T^{k-i}\right) J^{-1}=S^{i^{\prime}-i} \otimes S^{j^{\prime}-i} \otimes \cdots \otimes S^{k^{\prime}-i},
$$

сталкиваемся с ситуацией, когда несколько чисел в наборе $i^{\prime}-i, j^{\prime}-i, \ldots, k^{\prime}-i$ равны 0. Пусть для определенности $i^{\prime}-i=0$ и $j^{\prime}-i=0$, а другие разности отличны от нуля.

Пространство $L_{2} \otimes \mathbf{1} \otimes \cdots \otimes \mathbf{1}$ состоит из неподвижных точек оператора $I \otimes T^{j-i} \otimes$ $\cdots \otimes T^{k-i}$. Пространство $L_{2} \otimes L_{2} \otimes \mathbf{1} \otimes \cdots \otimes \mathbf{1}$ состоит из неподвижных точек оператора $S^{i^{\prime}-i} \otimes S^{j^{\prime}-i} \otimes \cdots \otimes S^{k^{\prime}-i}$. Поэтому

$$
J\left(L_{2} \otimes \mathbf{1} \otimes \cdots \otimes \mathbf{1}\right)=L_{2} \otimes L_{2} \otimes \mathbf{1} \otimes \cdots \otimes \mathbf{1}
$$

следовательно, автоморфизм $T$ изоморфен $S \times S$. Эта и подобные ей ситуации невозможны в силу леммы, которая завершает доказательство теоремы.

ЛЕмма 3. Если $T^{n_{k}} \rightarrow \mathscr{P}(T)$, то автоморфизм не может быть декартовой степенъю. 
ДоказАтельство. Пусть $T=S \otimes \cdots \otimes S\left(m>1\right.$ сомножителей). Так как $T^{n_{k}} \rightarrow$ $\mathscr{P}(T)$ влечет за собой $S^{n_{k}} \rightarrow \mathscr{P}(S)$, получим

$$
\mathscr{P}(S \otimes \cdots \otimes S)=\mathscr{P}(S) \otimes \cdots \otimes \mathscr{P}(S) .
$$

Левая и правая части равенства - выпуклые суммы различных операторов, коммутирующих с $S \otimes \cdots \otimes S$. Слева вес при операторе $\Theta \otimes \cdots \otimes \Theta$ равен $a$, справа $-a^{m}$. Поэтому $a=1$ или $a=0$. Если $a=0$, замечаем, что $a_{i}=a_{i}^{m}$ (вес оператора $S^{i} \otimes \cdots \otimes S^{i}$ в левой сумме равен его весу в правой сумме). Таким образом, равенство $(*)$ возможно только в том случае, когда $\mathscr{P}$ является мономом, что запрещено соглашением.

\section{СПИСОК ЦИТИРОВАННОЙ ЛИТЕРАТУРЫ}

[1] Д. В. Аносов, О спектральных кратностях в эргодической теории, Современные проблемы математики, 3, МИАН, М., 2003.

[2] А.М. Степин, "Спектральные свойства типичных динамических систем", Изв. АН СССР. Сер. матем., 50:4 (1986), 801-834.

[3] G.R. Goodson, "A survey of recent results in the spectral theory of ergodic dynamical systems", J. Dynamical Control Systems, 5:2 (1999), 173-226.

[4] A. A. Prikhod'ko, V. V. Ryzhikov, "Disjointness of convolutions for Chacon's automorphism", Colloq. Math., 84/85 (2000), 67-74.

[5] А. М. Степин, А. М. Еременко, "Неединственность включения в поток и обширность централизатора для типичного сохраняющего меру преобразования", Матем. сб., 195:12 (2004), 95-108.

[6] В.В. Рыжиков, "Типичность изоморфизма преобразований при изоморфизме их декартовых степеней”, Матем. заметки, 59:4 (1996), 630-632.

[7] А. М. Вершик, "Многозначные отображения с инвариантной мерой (полиморфизмы) и марковские процессы", Записки научных семинаров ЛОМИ, 72 (1977), 26-62.

\section{В. В. Рыжиков, А. Е. Троицкая}

Поступило Московский государственный университет 20.05.2005

им. М. В. Ломоносова

E-mail: vryz@mech.math.msu.su 